\title{
MODELOS DE CRESCIMENTO DA CANA-DE-AÇÚCAR E SUA PARAMETRIZAÇÃO - REVISÃO
}

Fábio Vale Scarpare ${ }^{1}$, Quirijn de Jong van Lier $^{2}$, Simone Toni Ruiz Corrêa ${ }^{3}$, Alexandre Hugo Cezar Barros ${ }^{4}$, Fábio Ricardo Marin ${ }^{5}$, Daniel Silveira Pinto Nassif ${ }^{6}$

\footnotetext{
${ }^{1}$ Pesquisador Laboratório Nacional de Ciência e Tecnologia do Bioetanol (CTBE)- fabio.scarpare@bioetanol.org.br

${ }^{2}$ Prof. Dr. Departamento de Engenharia de Biossistemas (ESALQ-USP)

${ }^{3}$ Doutoranda Fitotecnia (ESALQ-USP)

${ }^{4}$ Pesquisador Embrapa (CNPS)

${ }^{5}$ Pesquisador Embrapa (CNPTIA)

${ }^{6}$ Doutorando Engenharia de Sistemas Agrícolas (ESALQ-USP)
}

\section{RESUMO}

Em agronomia, modelos de simulação têm sido utilizados como ferramenta de pesquisa, possibilitando organizar o entendimento das respostas das plantas a diferentes ambientes e, consequentemente, predizer a produtividade das culturas. Modelos de crescimento de cultura usualmente possuem alto nível de complexidade apresentando grande número de parâmetros envolvidos com obtenções, em muitos casos, dependendo de grande número de dados experimentais. Diante da incontestável importância da cana-de-açúcar no cenário nacional o desenvolvimento e a adoção de ferramentas, como os modelos de previsão de produtividade usados na criação de cenários visando planejar e ou dimensionar a produção são fundamentais. Esse estudo tem como objetivo abordar os principais modelos utilizados para a simulação do crescimento da cana-de-açúcar bem como fornecer informações a respeito dos principais parâmetros comumente utilizados nesses modelos.

Palavras-chave: modelagem, parâmetro, simulação

\section{SUGARCANE CROP MODELLING AND PARAMETRIZATION - BRIEF REVIEW}

\section{ABSTRACT}

Simulation models have been used as a research tool in agriculture allowing organizing the understanding of the plant response under different environment, therefore predicting yield. Usually, crop growth models contain a high level of complexity, presenting a large number of parameters involved. In many cases, these parameters are obtained from a great number of experimental replicates done on the field. Due to the importance of sugarcane for the Brazilian economy, the development and use of crop modeling as a research tool in creating scenarios for planning the production are crucial. This study aimed to address the main models used to simulate the growth of sugarcane crop as well as to provide information about the main parameters commonly used in these models.

Keywords: modeling, parameter, simulation 


\section{INTRODUÇÃO}

\section{Modelagem de sistemas agrícolas}

Modelos podem ser traduzidos como objeto destinado a ser reproduzido por descrição ou a representação em pequena escala de algo com alta complexidade. No ramo da ciência, modelo pode ser definido como o conjunto de hipóteses sobre a estrutura ou o comportamento de um sistema físico pelo qual se pretende explicar ou prever, dentro de uma teoria científica, as propriedades do sistema (Ruiz-Corrêa et al., 2011).

Em agronomia, modelos de simulação têm sido utilizados como ferramenta de pesquisa, possibilitando organizar o entendimento das respostas das plantas a diferentes ambientes e, consequentemente, predizer a produtividade das culturas (Scarpare, 2011).

A rapidez em que o conhecimento no domínio da agricultura evolui associado à maior acessibilidade à informação tecnológica tem contribuído para um amplo desenvolvimento do número de modelos agrícolas ao longo das últimas décadas. Cheeroo-Nayamuth (1999) realizou extensa pesquisa entre os periódicos (Transaction of ASAE, Agricultural Systems, Agricultural and Forest Meteorology, Agronomy Journal e Field Crops Research) cujos trabalhos apresentassem no título os termos "modelagem e simulação" obtendo como resultado significativa evolução ao longo dos anos; de 82 estudos publicados na década de 70 para 270 estudos publicados até a década de 90. Esses dados corroboram a tendência de que os modelos aplicados em estudos em sistema agrícola estão sendo cada vez mais desenvolvidos e investigados.

Qualquer que seja a escala ou objetivo da modelagem, os modelos utilizados como suporte para a tomada de decisões geralmente contêm complexidade correspondente ao seu nível de detalhamento. Modelos de longo prazo (anuais ou maiores), e em grandes dimensões (planejamento nacional de safra), possivelmente são baseados em dados empíricos, geralmente oriundos de regressão entre o rendimento da cultura e outros fatores, como precipitação pluvial ou capacidade de armazenamento de água no solo (Argenton, 2006). Por outro lado, existem modelos baseados em dados provenientes de processos que ocorrem no dossel das culturas, operando em base de dados diária, alimentados com rigor mecanicista. A grande maioria dos modelos utilizados na simulação da biomassa, específicos para a cultura da cana-de-açúcar se enquadra nesse tipo de abordagem.

Esse estudo tem como objetivo abordar os principais modelos utilizados para a simulação do crescimento da cana-deaçúcar bem como fornecer informações a respeito dos principais parâmetros comumente utilizados.

\section{Modelos específicos para a cultura da cana-de-açúcar}

A modelagem na cultura da cana-deaçúcar data do início da década de 1970 e nesses quarenta anos, modelos com variados enfoques vêm sendo publicados na literatura científica. Restringindo a enorme gama de modelos existentes para aqueles em que o enfoque principal é o crescimento/desenvolvimento bem como o acúmulo de matéria seca, pode-se destacar inúmeros, tanto em âmbito nacional como internacional.

No Brasil, Ometto (1974) foi o pioneiro quando relacionou a estimativa da evapotranspiração potencial com o rendimento da cana-de-açúcar. Anos mais tarde, Machado (1981) apresentou um modelo matemático-fisiológico para simulação do acúmulo de matéria seca na 
cultura de cana-de-açúcar a partir de informações meteorológicas. Ainda na década de 80, Pereira e Machado (1986) descreveram o modelo SIMCANA (SIMulador matemático-fisiológico do crescimento diário de uma cultura de CANA-de-açúcar) onde três processos, normalmente estudados em separado (crescimento, fotossíntese e respiração), foram integrados. No SIMCANA, as taxas de fotossíntese, respiração e crescimento da cultura são simuladas em função das condições diárias de radiação solar global, temperatura máxima, mínima e umidade relativa do ar.

Villa Nova e Barbieri (1991) adaptaram o modelo matemático-fisiológico proposto por De Wit (1965) e o aplicaram para a cana-de-açúcar. Esse modelo relaciona a produtividade mensal de fitomassa com alguns elementos meteorológicos (temperaturas extremas do ar, fotoperíodo e insolação), aliados ao déficit hídrico ao longo do ciclo. Araujo (1993) aplicou a metodologia De Wit (1965), modificada por Villa Nova e Barbieri (1991), para estimar a produtividade potencial da cana-de-açúcar, com a finalidade de elaborar o zoneamento da produtividade potencial da cultura da cana-de-açúcar no Estado do Rio de Janeiro, tendo verificado que os valores estimados pelo modelo foram bem próximos aos valores de produtividade de cana irrigada.

Barbieri (1993) desenvolveu um modelo matemático-fisiológico de estimativa de produtividade da cana-de-açúcar. Esse modelo descreve o comportamento fisiológico da planta em resposta às condições ambientais, no qual elementos meteorológicos se relacionam com o desempenho da cultura, simulando o acúmulo potencial de matéria seca durante o ciclo.

Rojas (1998) empregou o modelo de Jensen (1968) para estimar os efeitos da deficiência hídrica na produtividade cana- de-açúcar no município de Araras, SP. Nesse modelo, o autor fez uma adaptação considerando que o valor do coeficiente de sensibilidade à deficiência hídrica na última fase de desenvolvimento da cultura tem pouca influência no resultado final. Desse modo, a produtividade real da cana-deaçúcar (colmo e açúcar) pode ser estimada com 4 a 6 meses de antecedência.

Teramoto (2003) avaliou o modelo matemático para estimativa de produtividade relativa de cana-de-açúcar baseado em parâmetros do solo (Levy, 1995) e o modelo da FAO (Food and Agriculture Organization of the United Nations) para estimativa do potencial produtivo (De Wit, 1965) para simular a produtividade da cana-de-açúcar mês a mês durante um ano. A metodologia usada no modelo da FAO emprega adaptações propostas por Barbieri e Tuon (1992).

Suguitani (2006) usou o modelo explanatório MOSICAS (Martiné, 2003) desenvolvido pelo Centre de Coopération Internationale en Recherche Agronomique pour le Développement (CIRAD) para simulação de crescimento de quatro variedades em condições edafoclimáticas do Estado de São Paulo apresentando resultados importantes, com valores simulados para massa seca da parte aérea e índice de área foliar aos observados em campo.

Scarpare (2011) adequou o modelo agrohidrológico SWAP/WOFOST (Van Dam et al., 2008) para a simulação de crescimento da massa seca da parte aérea da cana-de-açúcar com dados experimentais conduzidos na região canavieira paulista. Esse modelo foi aplicado às condições edafoclimáticas da região Centro-Sul brasileira apresentando bons resultados para a previsão da massa fresca de colmos industrializáveis em um leque de 25 cultivares.

Embora a contribuição nacional nesse assunto tenha sido importante, as escolas de modelagem de outros países 
tornaram-se mais reconhecidas devido ao fato de especificarem seus modelos a determinados fatores intrínsecos da cultura da cana-de-açúcar, além de organizarem seus modelos em linguagens computacionais. Dessa forma, além de facilitarem a utilização da ferramenta, promoveram a divulgação deles em âmbito mundial. Dentre os principais modelos referidos, destacam-se o APSIM-Sugarcane, CANEGRO, CANESIM e o QCANE.

$\mathrm{Na}$ África do Sul, Thompson (1976) desenvolveu um modelo relacional simples, precursor do atual modelo CANEGRO. O desenvolvimento do modelo CANEGRO originou-se em resposta às questões colocadas pelos produtores e cientistas da indústria açucareira Sul africana. A intenção foi modelar os processos fisiológicos mais relevantes de uma forma mecanicista e estabelecer uma base para a inclusão de outros processos que poderiam ser importantes para a indústria (Inman-Bamber, 2000). Esse modelo simula o crescimento da cana-de-açúcar usando informações dos elementos meteorológicos (radiação solar, precipitação pluvial, temperatura máxima e mínima do ar) e do balanço hídrico (capacidade de campo, ponto de murcha permanente e profundidade do perfil do solo). Com base em processo diário de crescimento da planta, CANEGRO inclui o desenvolvimento, a fenologia do dossel, o perfilhamento, o acúmulo de biomassa e sua partição, bem com o crescimento das raízes e o estresse hídrico. O impacto do estresse hídrico é simulado pelo ajuste dos diferentes processos da planta como a absorção de água, assimilação de $\mathrm{CO}_{2}$ e crescimento estrutural de acordo com as aproximações propostas por Singels e Bezuidenhout (2002). Nassif (2010) e Marin et al. (2011) utilizaram esse modelo adaptando-o para cultivares nacionais nas condições edafoclimáticas brasileiras com sucesso. Atualmente a versão mais recente do modelo
CANEGRO (Singels et al., 2008) foi incluída no programa DSSAT versão 4.5 (Decision Support System for Agrotechnology Transfer) (JONES et al., 2003).

O modelo CANESIM (Singels et al., 1999) desenvolvido na África do Sul, evoluiu de um modelo proposto para o controle de irrigação da cana-de-açúcar, o IRRICANE (Singels et al., 1998), com a inclusão de rotinas para simular a produtividade e também de um modelo para simular a área foliar da cultura. Este modelo foi desenvolvido com os mesmos conceitos básicos do modelo CANEGRO, entretanto é mais simples e requer menos parâmetros de entrada. Apesar de não ser tão robusto quanto seu precursor, o CANESIM pode ser utilizado por usuários tanto na indústria como no campo. O desenvolvimento do CANESIM ocorreu principalmente em função da demanda por informações no setor produtivo em suas atividades de planejamento. O modelo CANEGRO não atendia essa demanda porque exige uma quantidade excessiva de parâmetros de entrada, tornando-o inviável para usos aplicados e práticos que necessitam de respostas em curto prazo (Teramoto, 2003).

Na Austrália, Bull e Tovey (1974) iniciaram estudo com modelos para a canade-açúcar gerando posteriormente o APSIM-Sugarcane (Agricultural Production Systems simulator). Descrito por Keating et al. (1999), esse modelo opera em escala diária e relaciona o crescimento da cultura em função da radiação interceptada, a qual produz fotoassimilados que são particionados entre os diversos órgãos da planta. O processo fisiológico representado nesse modelo é governado pela radiação, temperatura ambiental e suprimento de água e nitrogênio $(N)$. O APSIM-Sugarcane simula o crescimento, o uso de água, acúmulo de $N$, teor de açúcar e matéria seca e fresca de colmos para o ciclo da canaplanta e socas. Sua validação foi realizada 
em amplo conjunto de dados espalhados em diversas regiões produtoras ao redor do mundo.

Outro modelo australiano, o QCANE (Liu \& Bull, 2001) foi desenvolvido para simular o crescimento e o acúmulo de açúcar nos colmos. Seu dinamismo baseia-se nos processos de desenvolvimento, incluindo o desenvolvimento do dossel, a fotossíntese, a respiração e a partição de carboidratos em órgãos vegetais para o crescimento. A respiração é ditada pelo desenvolvimento fenológico e ambientes em mudança. O balanço hídrico e o módulo de nitrogênio desse modelo são adaptados a partir do CERES-Maize (Jones \& Kiniry, 1986).

\section{Parametrização}

A parametrização é o processo de definição dos valores iniciais de entrada necessários para a estimação do modelo. Esse processo é essencial na modelagem matemática bem como na identificação de sistemas, uma vez que o estabelecimento de valores adequados para os parâmetros são requisitos básicos para que o modelo possa representar realisticamente o comportamento do sistema.

Os modelos de crescimento de cultura usualmente possuem alto nível de complexidade apresentando grande número de parâmetros envolvidos, para os quais a obtenção, em muitos casos, depende de grande volume de dados experimentais.

\section{Parâmetros relacionados com a assimilação de $\mathrm{CO}_{2}$}

Em modelos de simulação de crescimento como o CANEGRO, APSIM e AUSCANE, a abordagem para quantificar a assimilação diária de $\mathrm{CO}_{2}$ é por meio da eficiência do uso da radiação (RUE Radiation Use Efficiency). Robertson et al. (1996) relataram haver variação no valor
$R U E$ entre o ciclo da cana planta e cana soca em até $8 \%\left(1,72\right.$ e 1,59 $\left.\mathrm{g} \mathrm{MJ}^{-1}\right)$. Embora seja amplamente utilizada para simulação de muitas culturas, McWilliam et al. (1990) relatam que em modelos de simulação de crescimento de cana-de-açúcar baseados em $R U E$ para a assimilação diária de $\mathrm{CO}_{2}$, apresenta problemas quanto a simulação de biomassa e acúmulo de sacarose devido ao fraco componente biológico envolvido nessa abordagem.

Em outros modelos, como SWAP/WOFOST, QCANE e o SIMCANA, a assimilação diária de $\mathrm{CO}_{2}$ baseia-se na diferença entre fotossíntese, função da interceptação da radiação solar pelo dossel, com a respiração de manutenção, função do acúmulo de biomassa. Sendo assim, a relação entre fotossíntese e a luz absorvida pela folha de uma cultura pode ser descrita na forma de uma função que relaciona a taxa bruta de fotossíntese $\left(A_{p}\right.$ gross, $\left.\mathrm{kg} \mathrm{ha}^{-1} \mathrm{~h}^{-1}\right)$ sob condições ótimas, baseado na radiação incidente absorvida pelo dossel dessa cultura como proposta por Goudriaan (1982):

$$
A_{p \text { gross }}=A M A X\left(1-e^{\frac{\text { EFF PAR }}{A M A X}}\right)
$$

Esse tipo de curva é caracterizado por dois parâmetros: o ângulo de inclinação da curva, também denominado como eficiência do uso da luz $(E F F$, $\left.\mathrm{kg} \mathrm{ha} \mathrm{a}^{-1} \mathrm{~h}^{-1}\left(\mathrm{~J} \mathrm{~m}^{-2} \mathrm{~s}^{-1}\right)^{-1}\right)$ e a taxa máxima de assimilação de $\mathrm{CO}_{2}\left(A M A X, \mathrm{~kg} \mathrm{ha}^{-1} \mathrm{~h}^{-1}\right)$. PAR (Photossintetic Active Radiation) é a taxa de intensidade de radiação fotossinteticamente absorvível $\left(\mathrm{m}^{-2} \mathrm{~d}^{-1}\right)$.

De Wit et al. (1978) sugerem que para $E F F$ seja utilizado o valor de $0,48 \mathrm{em}$ plantas C4. Para a cana-de-açúcar valores da mesma ordem de grandeza foram determinados por Scarpare (2011) por meio de calibração automática para os ciclos canaplanta e soca $(0,49$ e 0,44$)$ respectivamente. 
Quanto a $A M A X$, em alta intensidade luminosa e concentração normal de $\mathrm{CO}_{2}$ seu valor geralmente encontra-se entre 25-80 $\mathrm{kg} \mathrm{ha}^{-1} \mathrm{~h}^{-1}$, podendo exceder e em espécies C4 (Penning De Vries et al., 1989). Rodrigues (1995) confirmou essa hipótese para a cana-de-açúcar, observando valores de taxa máxima de assimilação de $\mathrm{CO}_{2} \mathrm{em}$ até $100 \mathrm{~kg} \mathrm{ha}^{-1} \mathrm{~h}^{-1}$. Esse parâmetro depende fortemente das propriedades das plantas como a área foliar específica (SLA - Specific Leaf Area, ha kg-1) e das condições ambientais, como a temperatura (Penning De Vries et al., 1989).

Para o parâmetro da área foliar específica (SLA) Machado (1981) determinou o valor de 0,0010 ha $\mathrm{kg}^{-1}$ da cana-de-açúcar variedade NA 56-79 em Piracicaba-SP. Silva et al. (2005) calcularam a área foliar específica ao longo do ciclo da cana-soca em 5 cultivares (RB 845197, RB 845210, RB 855113, RB 855536 e RB 928064) na região noroeste do Paraná observando que os comportamentos entre as cultivares estudadas foram bastante semelhantes. Os maiores valores reportados foram obtidos no início do ciclo da cultura, devido ao acúmulo de reservas nas folhas decrescendo com o desenvolvimento do ciclo $\quad(0,0035 ; \quad 0,0025 ; 0,0018 ; 0,0015$;

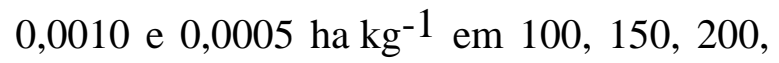
250, 300 e 350 dias após o corte). Isso sugere que a cana-de-açúcar transloca suas reservas das folhas para outras partes da planta, principalmente para os colmos.

Com relação à longevidade da folha no dossel da planta, Machado et al. (1982) verificaram que a longevidade de uma folha fotossinteticamente ativa pode variar de 30 a 150 dias, em função da idade da planta e condições climáticas. Irvine (1983) observou que essa longevidade é maior no período de maior crescimento vegetativo e que nessa fase chegava a ser de 150 dias. Cock (2001) encontrou valor similar, com duração de 140 dias quando os perfilhos estavam com 4 meses de idade. Dillewijn (1952) no Havaí verificou que a duração média de uma folha variava de 60 a 75 dias. Van Heemst (1986) determinou, para a cana-de-açúcar, valor do parâmetro SPAN (quantidade máxima em dias pela qual a folha pode viver em temperatura constante de $35^{\circ} \mathrm{C}$ ) de 60 dias.

A maneira como a área foliar é distribuída verticalmente na copa também influencia a proporção de luz incidente que penetra no dossel e, consequentemente, a fotossíntese (Suguitani, 2006). Parte da radiação fotossinteticamente ativa $(P A R)$ é refletida pela copa. $\mathrm{O}$ coeficiente de reflexão $(\rho)$ está em função da elevação solar, distribuição angular das folhas e propriedades das folhas quanto à transmissão e reflexão. A fração não refletida, (1- $\rho$ ) é potencialmente disponível para a absorção pelo dossel. Contudo, o fluxo radiativo decresce de forma exponencial no sentido do interior do dossel e é descrito por Spitters et al. (1989) baseado na lei de Beer, conforme a equação:

$I_{L}=(1-\rho) * I_{0} * e^{-k * I A F}$

onde $I_{L}$ é o fluxo de radiação líquida na camada $L$ do dossel, $I_{0}$ é o fluxo de radiação incidente no topo do dossel $\left(\mathrm{W} \mathrm{m}^{-2}\right), I A F$ é o índice de área foliar $\left(\mathrm{m}^{2} \mathrm{~m}^{-2}\right)$ e $k$ o coeficiente de extinção da radiação solar (direta ou difusa).

A extinção da fração da luz fotossinteticamente ativa (PAR) ocorre com diferentes coeficientes: 0,50 para radiação direta e 0,72 para a radiação difusa quando o ângulo de distribuição é esférico, ou seja, faces da folha distribuída como a superfície de um globo. Dessa forma, o coeficiente médio de extinção é de aproximadamente 0,60 para dossel com folhas eretas e 0,80 para dossel com folhas horizontais (Goudriaan, 1977). Muchow et al. (1994) e Robertson et al. (1996) determinaram o valor de 0,38 para o coeficiente de extinção 
da radiação em cultivo de cana-de-açúcar na Austrália. Para valores do índice de área foliar maiores que 1,0 Barbieri (1993) chegou ao valor de $k$ igual a 0,58 enquanto que Bernardes e Lima (2000) determinaram seu valor na faixa de 0,70 . Zhou et al. (2003) determinaram o coeficiente de extinção da radiação fotossinteticamente ativa ao longo do ciclo da cana-planta no Zimbábue não observando diferenças significativas ao nível de $5 \%$ de probabilidade entre os 4 cultivares estudados (Tabela 1).

Tabela 1. Coeficiente de extinção para as cultivares ZN6, ZN7, N14 e NCo 376 ao longo do ciclo da cana planta no Zimbábue

\begin{tabular}{ccccc}
\hline \hline Dias após o plantio & ZN6 & ZN7 & N14 & NCo 376 \\
\hline 87 & 0,447 & 0,478 & 0,471 & 0,482 \\
95 & 0,430 & 0,424 & 0,409 & 0,426 \\
102 & 0,493 & 0,497 & 0,488 & 0,502 \\
109 & 0,559 & 0,571 & 0,582 & 0,584 \\
116 & 0,675 & 0,640 & 0,597 & 0,662 \\
124 & 0,539 & 0,699 & 0,537 & 0,562 \\
131 & 0,588 & 0,607 & 0,571 & 0,609 \\
Média & 0,533 & 0,559 & 0,522 & 0,547 \\
\hline \hline
\end{tabular}

Fonte: Zhou et al. (2003)

Os dados desse estudo mostraram que houve aumento do coeficiente de extinção para todas as cultivares analisadas ao longo de desenvolvimento do ciclo. Isto está de acordo com as tendências encontradas por Inman-Bamber (1994). Na Austrália, Park et al. (2005) determinaram valor médio de $k$ em 0,40 estudando 5 cultivares no ciclo da cana planta e soca. Os autores relataram que valores ligeiramente superiores de $k$ foram calculados no ciclo da soca, associando-os à produção precoce de folhas e ao rápido fechamento do dossel da cultura.

\section{Respiração}

O termo respiração tem sido empregado como a perda de $\mathrm{CO}_{2}$ ou de matéria seca pela planta. No entanto, a respiração é o processo pelo qual, compostos altamente energéticos são sintetizados, a partir dos carboidratos produzidos na fotossíntese. Assim, a respiração pode ser dividida, teoricamente, em respiração de crescimento e de manutenção (McCree, 1974).

A respiração de manutenção é usada para manter a estrutura da planta. A energia de manutenção difere entre os diferentes componentes da planta, sendo controlada pela temperatura ambiente e pela senescência da planta. Geralmente, é calculada em função da matéria seca total acumulada pela planta e temperatura média do ar.

Penning de Vries et al. (1979) estimaram que o consumo de carboidratos 
pela respiração de manutenção durante o ciclo de crescimento de uma cultura pode atingir entre 15 a $30 \%$ da assimilação. Penning de Vries et al. (1989) relataram que para as folhas, os valores da taxa da respiração de manutenção medida em plantas cultivadas a campo são 0,03-0,08 g $\mathrm{CO}_{2}$ por grama de matéria seca por dia a $20^{\circ} \mathrm{C}$ (para as culturas de clima temperado) ou a $30^{\circ} \mathrm{C}$ (para culturas tropicais). Em outros órgãos das plantas, Penning de Vries et al. (1989) descreveram taxas entre 0,005$0,090\left(\mathrm{~g} \mathrm{~g}^{-1} \mathrm{~d}^{-1}\right)$, sendo que para as raízes e caules de plantas anuais as taxas são de 0,015 e 0,010 , respectivamente.

Para a cana-de-açúcar, Liu e Bull (2001) utilizaram dados de Glover (1973) para desenvolver equações relacionando a respiração de manutenção em diferentes órgãos da planta sob diferentes temperaturas. Como resultados, os autores concluíram que a taxa da respiração de manutenção varia significativamente entre folhas e colmos $\left(0,0157\right.$ e $0,00498 \mathrm{~g} \mathrm{~g}^{-1} \mathrm{~d}^{-1}$ a $30^{\circ} \mathrm{C}$, respectivamente) e em diferentes temperaturas $\left(0,00276\right.$ e $0,00498 \mathrm{~g} \mathrm{~g}^{-1} \mathrm{~d}^{-1}$ para colmos a 20 e $30^{\circ} \mathrm{C}$, respectivamente). A mudança nas frações de massa dos órgãos da planta ao longo do tempo também afeta significativamente o total da taxa respiratória da planta, por exemplo, 0,00972 a 0,00843 g $\mathrm{g}^{-1} \mathrm{~d}^{-1}$ a $30^{\circ} \mathrm{C}$ para canaviais com 60 a $70 \%$ de massa seca da parte aérea proveniente de colmos. O modelo CANEGRO (Singels; Bezuidenhout, 2002) usa um valor constante para a respiração de manutenção para todos os órgãos de $0,004 \mathrm{~g} \mathrm{~g}^{-1} \mathrm{~d}^{-1}$ independente da temperatura. A segunda versão desse modelo aborda a respiração de manutenção baseado em Liu e Bull (2001).

A temperatura afeta de forma direta a taxa de respiração de manutenção e seu incremento relativo na taxa da respiração com aumento da temperatura em $10 \mathrm{C}$ é representado em alguns modelos pelo conceito biológico Q10. Para a cana-deaçúcar, Inman-Bamber (1991) determinou o valor de $Q 10$ igual a 1,68 enquanto que Waldron et al. (1967) verificaram que a mesma apresenta $Q 10$ de 1,1 na faixa de temperatura que vai de 8 a $34^{\circ} \mathrm{C}$.

A respiração de crescimento é definida como a evolução do $\mathrm{CO}_{2}$ resultante do processo de crescimento. A eficiência e a respiração de crescimento são dois aspectos do mesmo processo. Assim a respiração de crescimento está relacionada com a eficiência de conversão do carboidrato assimilado em matéria seca, além do dispêndio de energia pelo seu transporte do órgão de origem para o órgão de crescimento, sendo calculada com base nos fatores de conversão de cada órgão, que estão em função da sua composição química (Penning De Vries et al., 1989).

Penning de Vries (1975) demonstrou que, a partir do conhecimento da composição química de biomassas e dos processos bioquímicos envolvidos em sua síntese, foi possível estimar a eficiência de conversão dos assimilados em biomassa ( $\mathrm{kg}$ $\mathrm{kg}^{-1}$ ). Para essa finalidade o autor calculou a eficiência de conversão da glicose em cada um dos principais componentes da biomassa (proteínas, aminoácidos, celulose, lignina, lipídeos e etc.), ou seja, a massa de cada composto formado a partir de $1 \mathrm{~g}$ de glicose. Dessa forma, com o conhecimento de conversão de cada composto, bem como da composição química das plantas em estudo, pode-se estimar o valor médio da eficiência global de conversão do processo construtivo da respiração (Machado, 1981).

Exemplos de fatores de conversão para diferentes constituintes químicos e valores médios do fator de conversão para a parte aérea (folha e colmos) da cana-deaçúcar constam nas Tabelas 2 e 3. 
Tabela 2. Características da biossíntese de cinco grupos de compostos orgânicos: (A) gastos com biossíntese ( $\mathrm{g}$ de carboidrato por $\mathrm{g}$ do produto), (B) gastos com transporte ( $\mathrm{g}$ de carboidrato por $\mathrm{g}$ do produto), $(\mathrm{C}=\mathrm{A}+\mathrm{B})$ gastos de conversão ( $\mathrm{g}$ de carboidrato por $\mathrm{g}$ do produto) e $(\mathrm{D}=1 / \mathrm{C})$ (g produto por g de carboidrato).

\begin{tabular}{lcccc}
\hline \hline \multicolumn{1}{c}{ Composto } & $\mathrm{A}$ & $\mathrm{B}$ & $\mathrm{C}$ & $\mathrm{D}=\mathrm{CVF}$ \\
\hline Gordura & 3,030 & 0,159 & 3,189 & 0,31 \\
Lignina & 2,119 & 0,112 & 2,231 & 0,45 \\
Proteínas & 1,824 & 0,096 & 1,920 & 0,52 \\
Carboidratos & 1,211 & 0,064 & 1,275 & 0,78 \\
Ácidos Orgânicos & 0,906 & 0,048 & 0,954 & 1,05 \\
Minerais (K, Ca, P) & 0,000 & 0,120 & 0,120 & 8,33 \\
\hline \hline
\end{tabular}

CVF: Fator de conversão

Fonte: Penning de Vries et al. (1989)

Tabela 3. Composição química (valores médios para a parte aérea da cana-de-açúcar), em carboidrato $\left(\mathrm{CH}_{2} \mathrm{O}, \mathrm{kg} \mathrm{kg}-1\right.$ ), proteína ( $\mathrm{PRO}, \mathrm{kg} \mathrm{kg}-1$ ), gordura (GOR, kg kg-1), lignina (LIG, kg kg-1), ácidos orgânicos (AOR, kg kg-1) e minerais (MIN, kg kg-1), e fator de conversão (CVF, g g-1 - g do produto por g de carboidrato).

\begin{tabular}{cccccccc}
\hline & $\mathrm{CH}_{2} \mathrm{O}$ & PRO & GOR & LIG & AOR & MIN & CVF \\
\hline Parte aérea & 0,57 & 0,07 & 0,02 & 0,22 & 0,06 & 0,06 & 0,70 \\
\hline \hline
\end{tabular}

Fonte: Penning de Vries et al. (1983)

Penning de Vries et al. (1989) relataram que a eficiência da conversão de carboidrato nas culturas geralmente varia de 68 a 86\%, sendo que as leguminosas possuem, em média, taxa $10 \%$ menor devido às custas de redução do nitrogênio.

Machado (1981) estimou a composição química da cana-de-açúcar, cultivar NA 56-79 combinando os valores da composição química com os valores de conversão da glicose estimando valor de 1,27 g de glicose para $1 \mathrm{~g}$ de produção de matéria seca. Consequentemente, a eficiência de conversão de biomassa foi de $0,79 \mathrm{~kg} \mathrm{~kg}^{-1}$.

A respiração de crescimento no modelo QCANE (Liu \& Bull, 2001) é calculada pela multiplicação da taxa de crescimento pelo fator $0,33 \quad \mathrm{~g} \quad \mathrm{~g}^{-1}$ determinado por Thornley \& Johnson (1990). O modelo CANEGRO (Singels et al., 2008) utiliza valor de respiração de crescimento de 0,242 $\mathrm{g} \mathrm{g}^{-1}$ (Inman-Bamber, 1991).

\section{Partição de fotoassimilados}

A partição dos fotoassimilados em modelos de simulação de crescimento de cana-de-açúcar é um processo de grande importância devido ao fato de poder quantificar o volume de matéria seca destinada à usina além do teor de sacarose nela contida. É conhecido que a temperatura e o estresse hídrico afetam as frações de partição (Liu \& Bull, 2001; Singels \& 
Inman-Bamber, 2002), contudo, nem todos os modelos específicos que simulam o crescimento da cana-de-açúcar levam esses fatores em consideração.

O APSIM-Sugarcane (Keating et al., 1999) calcula a massa seca das raízes como sendo uma fração da massa seca da parte aérea, variando de 0,3 na emergência a 0,2 para o florescimento. Para a parte aérea, esse modelo destina $70 \%$ dos fotoassimilados para os colmos.

O CANEGRO (Singels et al., 2008) particiona os fotoassimilados utilizando uma função não linear ao longo do desenvolvimento da cultura. No início do desenvolvimento, as raízes são os principais destinos dos assimilados correspondendo com 0,95 , enquanto que a fração destinada para a parte aérea no fim do desenvolvimento corresponde a 0,88. O modelo utiliza as aproximações de Singels et al., (2005) para a inclusão do efeito da temperatura na fração de partição da parte aérea.

O modelo QCANE (Liu \& Bull, 2001) incluiu equações que descrevem o efeito da temperatura na partição. Seu maior efeito foi para plantas em estádio de maturação em temperaturas abaixo de $16^{\circ} \mathrm{C}$. A fração de partição de colmos aumentou de 0,41 a $16^{\circ} \mathrm{C}$ para 0,61 a $10^{\circ} \mathrm{C}$.

Singels et al. (2005) revisaram diversos trabalhos sobre esse assunto concluindo que a grande variabilidade da fração de partição de colmos encontrada na literatura se deve a resultados provenientes de diferentes métodos de medida e determinações de biomassa (Tabela 4).

Tabela 4. Fração destinada aos colmos de cana-de-açúcar dentre a massa seca da parte aérea.

\begin{tabular}{lll}
\hline \hline Referência & Fração de colmos & Observação \\
\hline Robertson et al. (1996) & 0,80 & Cultivar australiano \\
Rostron (1972) & 0,65 & \\
Dillewijn (1952) & 0,59 & \\
Evensen et al. $(1997)$ & 0,66 & Cultivar havaiano \\
Thompson (1988) & 0,69 & Dependente do ciclo \\
Thompson (1991) & $0,64-0,73$ & \\
Inman-Bamber et al. (2002) & 0,85 & Cultivar sul-africano \\
Inman-Bamber e Thompson (1989) & 0,70 & $\begin{array}{l}\text { Valor máximo obtido por cultura } \\
\text { Singels } \text { et al. } \text { (2000) }\end{array}$ \\
\end{tabular}

Fonte: Singels et al. (2005) 


\section{CONCLUSÃO}

Com o desenvolvimento da tecnologia, programas computacionais e melhoria de técnicas de modelagem, a modelagem agrícola está se tornando uma ferramenta de pesquisa cada vez mais comum. Desde as primeiras abordagens no início da década de 1970 até os dias atuais observa-se expressiva evolução nos mecanismos abordados nos modelos de simulação do crescimento da cana-de-açúcar bem como aumento no número de grupos de pesquisadores envolvidos nesses processos.

Com relação à parametrização, devido à grande diversidade de cultivares, ambiente e formas de análise de dados experimentais recomenda-se critério na utilização de valores dos parâmetros de entrada nos modelos de simulação. Além do cuidado do usuário em utilizar valores compatíveis na ordem de grandeza é fundamental a realização da análise de sensibilidade do modelo a esses parâmetros.

A modelagem é uma ferramenta poderosa de auxílio à pesquisa. Embora bons resultados tenham sido relatados com a utilização de modelos mecanísticos na previsão de safra para a cana-de-açúcar, o modelador deve estar atento e ciente de suas limitações. Sendo assim, a utilização de modelo não substitui a experimentação a campo, mas sim deve auxiliar fazendo com que os recursos sejam melhor alocados e, assim, a qualidade das informações sejam mais relevantes à pesquisa.

\section{REFERÊNCIAS BIBLIOGRÁFICAS}

ARAUJO, M. I. M. 1993. Zoneamento da produtividade potencial climática da cana-de-açúcar no Estado do Rio de Janeiro. 84 p. Dissertação (Mestrado em Fitotecnia) - Escola Superior de Agricultura "Luiz de Queiroz", Universidade de São Paulo, Piracicaba.
ARGENTON, P. E. 2006. Influências das variáveis edafoclimáticas e de manejo no rendimento de variedades de canade-açúcar (Saccharum spp.) na região de Piracicaba, São Paulo. 109 p. Dissertação (Mestrado em Fitotecnia) Escola Superior de Agricultura "Luiz de Queiroz", Universidade de São Paulo, Piracicaba.

BARBIERI, V. 1993.Condicionamento climático da produtividade potencial da cana-de-açúcar (Saccharum spp.): um modelo matemático-fisiológico de estimativa. 142 p. Tese (Doutorado em Agrometeorologia) - Escola Superior de Agricultura "Luiz de Queiroz", Universidade de São Paulo, Piracicaba.

BARBIERI, V.; TUON, R. L. 1992. Metodologia para estimativa da produção potencial de algumas culturas. Piracicaba: ESALQ, Departamento de Física e Meteorologia, $17 \mathrm{p}$.

BERNARDES, M. S.; LIMA, S. F. F. 2000. Densidade populacional, disposição e direção de linhas de plantio, manejo da copa: a luz como fator essencial. Piracicaba: ESALQ, 55 p.

BULL, T. A.; TOVEY, D. A. 1974. Aspects of modeling sugarcane growth by computer simulation. In: INTERNATIONAL SOCIETY SUGARCANE TECHNOLOGISTS, 15., Durban. Proceedings ... Durban: ISSCT, p. 1021-1032.

CHEEROO-NAYAMUTH, F. B. 1999. Crop modelling / simulation: an overview. Réduit: Maurits Sugar Industry Research Institute, p. 11-26.

COCK, J. H. 2001. Sugarcane growth and development. In: SIMPÓSIO INTERNACIONAL DE FISIOLOGIA DA CANA-DE-AÇÚCAR, 1., 2001, Piracicaba. Material preliminar... Piracicaba: STAB, 1 CD-ROM. 
DE WIT, C. T. 1965. Photosyntesis of leaf canopies. Agricultural Research Report, 663. Wageningen: Pudoc, 57 p.

DE WIT, C. T. 1978. Simulation of assimilation, respiration and transpiration of crops. Wageningen: Pudoc, 140 p. (Simulation Monographs).

DILLEWIJN, C. 1952. Botany of sugar cane. Walthen: Chronica Botanica, 359 p.

EVENSEN, C. I.; MUCHOW, R. C.; ElSWAIFY, S. A.; OSGOOD, R. V. 1997. Yield accumulation in irrigated sugarcane: I. Effect of crop age and cultivar. Agronomy Journal, Madison, v. 89 , p. $638-646$.

GOUDRIAAN, J. 1977. Crop micrometeorology: a simulation study. Wageningen: Pudoc, 249 p. (Simulation Monographs).

GOUDRIAAN, J. 1982. Potential production processes. In: PENNING DE VRIES, F.W.T.; VAN LAAR, H.H. (Ed.): Simulation of plant growth and crop production. Wageningen: Pudoc, p. 98-113. (Simulation Monographs).

GLOVER, J. 1973. The dark respiration of sugarcane and the loss of photosynthate during growth of the crop. Annals of Botany, London, v. 37, p. 845-852

INMAN-BAMBER, N. G. 1991. A growth model for sugarcane based on a simple carbon balance and CERES-Maize water balance. South African Journal of Plant and Soil, Pretoria, v. 8, p. 93-99.

INMAN-BAMBER, N. G. 1994. Temperature and seasonal effects on canopy development and light interception of sugarcane. Field Crops Research, Amsterdam, v. 36, n. 1, p. 4151.

INMAN-BAMBER, N. G. 2000. History of the CANEGRO model. Proceedings of International CANEGRO Workshop, Mount Edgecombe, South Africa, p. 4-7

INMAN-BAMBER, N. G.; THOMPSON, G.D. 1989. Models of dry matter accumulation by sugarcane. Proceedings of the South African Sugar Technologists Association, Durban, v. 63, p. 212-216.

INMAN-BAMBER, N. G.; MUCHOW, R. C.; ROBERTSON, M. J. 2002. Dry matter partitioning of sugarcane in Australia and South Africa. Field Crops Research, Amsterdam, v. 76, p. 71-84.

IRVINE, J. E. 1983. Sugarcane. In: SYMPOSIUM ON POTENCIAL PRODUCTIVITY OF FIELD CROPS UNDER DIFFERENT ENVIRONMENT, 1980, Los Banos. Proceedings... Los Banos: IRRI, p. 361381.

JENSEN, M. E. 1968. Water consumptions by agricultural plant growth. New York: Academic Press, v. 2, p. 1-22.

JONES, C. A.; KINIRY, J. R. 1986. CERES-Maize: a simulation model of maize growth and development. Texas: A\&M University Press, $194 \mathrm{p}$.

JONES, J. W.; HOOGENBOOM, G.; PORTER, C. H.; BOOTE, K. J.; BATCHELOR, W. D.; HUNT, L. A.; WILKENS, P. W.; SINGH, U.; GIJSMAN, A. J.; RITCHIE, J. T. 2003. The DSSAT cropping system model. European Journal of Agronomy, Amsterdam, v. 18, p. 235-265.

KEATING, B. A.; ROBERTSON, M. J.; MUCHOW, R. C.; HUTH, N. I. 1999. Modelling sugarcane production systems. 1. Development and performance of the sugarcane module. Field Crops Research, Amsterdam, v. 61, n. 3, p. 253-271.

LEVY, M. C. T. C. 1995. Avaliação de cenários da produção agrícola visando à sustentabilidade do uso das terras de Piracicaba (SP). 104 p. Dissertação (Mestrado em Fitotecnia) - Escola Superior de Agricultura "Luiz de Queiroz", Universidade de São Paulo, Piracicaba. 
LIU, D. L.; BULL, T. A. 2001. Simulation of biomass and sugar accumulation in sugarcane using a process-based model. Ecological Modelling, Amsterdam, v. 144, p. 181-211.

MACHADO, E. C. 1981. Um modelo matemático-fisiológico para simular o acúmulo de material seca na cultura da cana-de-açúcar (Saccharum spp.). 115 p. Dissertação (Mestrado em Biologia Vegetal) - Instituto de Biologia, Universidade Estadual de Campinas, Campinas

MACHADO, E. C.; PEREIRA, A. R.; FAHL, J. I.; ARRUDA, J. V.; CIONE, J. 1982. Índices biométricos de duas variedades de cana-de-açúcar. Pesquisa Agropecuária Brasileira, Brasília, v. 17, n. 9, p. 1323-1329.

MARIN, F. R.; JONES, J. W.; ROYCE, F.; SUGUITANI, C.; DONZELLI, J. L.; PALLONE FILHO, W. J.; NASSIF, D. S. P. 2011. Parameterization and evaluation of predictions of DSSAT/CANEGRO for Brazilian sugarcane. Agronomy Journal, Madison, v. 103, p. 297-303.

MARTINÉ, J. F. 2003. Modélisation de la production potentielle de la canne à sucre em zone tropicale, sous conditions thermiques et hydriques contrastées. Applications du modéle. 130 p. Thése (Docteur) - Institut National Agronomique Paris-Gragnon, Paris.

McCREE, K. J. 1974. Equations for the rate of dark respiration of white clover and grain sorghum, as functions of dry weight, photosynthetic rate and temperature. Crop Science, Madison, v. 14, p. 509-514.

McWILLIAM， J. R.; KINGSTON, K.; HAMMER, G. L. 1990. The role of AUSCANE as a tool in research and development for the Australian sugar industry. Report on review of AUSCANE model to BSES.P. 12.
MUCHOW, R. C.; SPILLMAN, M. F.; WOOD, A. W.; THOMAS, M. R. 1994. Radiation interception and biomass accumulation in a sugarcane crop grown under irrigation tropical conditions. Australian Journal of Agricultural Research, Victoria, v. 45, p. 37-49.

NASSIF, D. S. P. 2010. Parametrização e avaliação do modelo DSSAT/CANEGRO para variedades brasileiras de cana-de-açúcar. 2010. 92 p. Dissertação (Mestrado em Física do Ambiente Agrícola) - Escola Superior de Agricultura "Luiz de Queiroz", Universidade de São Paulo, Piracicaba.

OMETTO, J. C. 1974. Equação para a estimativa de evapotranspiração potencial, sua aplicação no cálculo das necessidades hídricas e do rendimento agro-industrial da cana-de-açúcar na região de Piracicaba - SP. 1974. 129 p. Tese (Livre Docência) - Escola Superior de Agricultura "Luiz de Queiroz", Universidade de São Paulo, Piracicaba.

PARK, S. E.; ROBERTSON, M.; INMANBAMBER, N. G. 2005. Decline in the growth of a sugarcane crop with age under high input conditions. Field Crops Research, Amsterdam, v. 92, p. 305-320.

PENNING DE VRIES, F. W. T. 1975. The cost of maintenance processes in plant cells. Annals of Botany, London, v. 39, p. 77-92.

PENNING DE VIRES, F. W. T.; WITLAGE, J. M.; KREMER, D. 1979. Rates of respiration and of increase in structural dry matter in young wheat, ryegrass and maize plants in relation to temperature, to water stress and to their sugar content. Annals of Botany, London, v. 44, p. 595-609.

PENNING DE VRIES, F. W. T.; VAN LAAR, H. H.; CHARDON, M. C. M. 1983. Bioenergetics of growth of seeds, fruits, and storage organs. In: SMITH, W.H.; BANATA, S.J. (Ed.). Potential 
productivity of field crops under different environments. Los Banos: International Rice Research Institute, $\mathrm{p}$. 37-59.

PENNING DE VRIES, F. W. T.; JANSEN, D. M.; TEN BERGE, H. F. M.; BAKEMA, A. 1989. Simulation of ecophysiological processes of growth in several annual crops. Wageningen: Pudoc, $271 \mathrm{p}$.

PEREIRA, A. R.; MACHADO, E. C. 1986. Um simulador dinâmico do crescimento de uma cultura de cana-de-açúcar. Bragantia, Campinas, v. 45, p. 107-122

ROBERTSON, M. J.; WOOD, A. W.; MUCHOW, R. C. 1996. Growth of sugarcane under high input conditions in tropical Australia. I. Radiation use, biomass accumulation and partitioning. Field Crops Research, Amsterdam, v. 48, p. 11-25.

RODRIGUES, J. D. 1995. Fisiologia da cana-de-açúcar. Botucatu: UNESP, 75 p.

ROJAS, J. S. D. 1998. Modelo agrometeorológico para estimativa dos efeitos de deficiência hídrica na produtividade agro-industrial da cana-de-açúcar. 1998. 74 p. Dissertação (Mestrado em Agrometeorologia) Escola Superior de Agricultura "Luiz de Queiroz", Universidade de São Paulo, Piracicaba

ROSTRON, H. 1972. The effects of age and time of harvest on the productivity of irrigated sugarcane. South African Sugar Technologists Association, Durban, v. 46, p. 142-150.

RUIZ-CORRÊA, S. T.; DOURADO-NETO, D. LORENÇONI, R.; SCARPARE, F. V.; VIVIAN, R.; RUIZ, E. T. 2011. Aplicações e limitações da modelagem em agricultura - Revisão. Revista de Agricultura, Piracicaba, v. 86, p. 1-13.

SCARPARE, F. V. 2011. Simulação de crescimento da cana-de-açúcar pelo modelo

agrohidrológico
SWAP/WOFOST. 2011. 163 p. Tese (Doutorado em Física do Ambiente Agrícola) - Escola Superior de Agricultura "Luiz de Queiroz", Universidade de São Paulo, Piracicaba.

SINGELS, A.; KENNEDY, A. J.; BEZUIDENHOUT, C. N. 2000. The effect of water stress on sugarcane biomass accumulation and partitioning. Proceedings of the South African Sugar Technologists Association, Durban, v. 74, p. 169-172.

SINGELS, A.; INMAN-BAMBER, N. G. 2002. The responses of sugarcane to water stress: preliminary results from a collaborative project. Proceedings of the South African Sugar Technologists Association, Durban, v. 76, p. 240-244.

SINGELS, A.; BEZUIDENHOUT, C. N. 2002. A new method of simulating dry matter partitioning in the Canegro sugarcane model. Field Crops Research, Amsterdam, v. 78, p. 151164.

SINGELS, A.; DONALDSON, R. A.; SMIT, M. A. 2005. Improving biomass production and partitioning in sugarcane: theory and practice. Field Crops Research, Amsterdam, v. 92, p. 291303.

SINGELS, A.; JONES, M.; VAN DEN BERG, M. 2008. DSSAT v.4.5 DSSAT/CANEGRO: sugarcane plant module; scientific documentation. Mount Edgecombe: International Consortium for Sugarcane Modeling; South African Sugarcane Research Institute, $34 \mathrm{p}$.

SINGELS, A.; KENNEDY, A. J.; BEZUIDENHOUT, C. N. 1998. IRRICANE: a simple irrigation scheduling procedure for sugarcane using automatic weather stations. Proceedings of the South African Sugar Technologists Association, Durban, v. 72, p. 117-122. 
SINGELS, A.; KENNEDY, A. J.; BEZUIDENHOUT, C. N. 1999. Weather based decision support through the Internet for the agronomic management of sugarcane. Proceedings of the South African Sugar Technologists Association, Durban, v. 73, p. 30-32.

SILVA, D. K. T.; DAROS, E.; ZAMBOM, J. L. C.; WEBER, H.; IDO, O. T.; ZUFELLATTO-RIBAS, K. C.; KOEHLER, H. S.; OLIVEIRA, R. A. 2005. Análise de crescimento em cultivares de cana-de-açúcar em cana soca no noroeste do Paraná na safra 2002/2003. Scientia Agraria, Curitiba, v. 6, p. 47-53.

SPITTERS, C. J. T.; VAN KEULEN, H.; VAN KRAALINGEN, D. W. G. 1989. A simple and universal crop growth simulator: SUCROS87. In: RABBINGE, R.; WARD, S.A.; VAN LAAR, H.H. (Ed.). Simulation and systems management in crop protection. Wageningen: Pudoc, p. 147-181. (Simulation Monographs).

SUGUITANI, C. 2006. Entendendo o crescimento e produção da cana-deaçúcar: avaliação do modelo Mosicas. 2006. 60 p. Tese (Doutorado em Fitotecnia) - Escola Superior de Agricultura "Luiz de Queiroz", Universidade de São Paulo, Piracicaba

TERAMOTO, E. R. 2003. Avaliação e aplicação de modelos de estimativa de produção de cana-de-açúcar (Saccharum spp.) baseados em parâmetros do solo e do clima. 2003. 86 p. Tese (Doutorado em Fitotecnia) Escola Superior de Agricultura "Luiz de Queiroz", Universidade de São Paulo, Piracicaba.

THOMPSON, G. D. 1976. Water use by sugarcane. South African Sugar Journal, Durban, v. 60, p. 593-600.

THOMPSON, G. D. 1988. Comparisons of the growth of plant and first ratoon crops of sugarcane at Pongola. Proceedings of the South African Sugar Technologists Association, Durban, v. 62, 180-184.

THOMPSON, G. D. 1991. The growth of sugarcane cultivar N14 at Pongola. Mount Edgecombe: South African Sugarcane Research Institute, 538 p. (Research Report, 7).

THORNLEY, J. H. M.; JOHNSON, I. R. 1990. Plant and crop modelling. Oxford: Oxford University Press, 669 p.

VAN DAM, J. C.; GROENENDIJK, P.; HENDRIKS, R. F. A.; KROES, J. G. 2008. Advances of modeling water flow in variably saturated soils with SWAP. Vadose Zone Journal, Madison, v. 7, n. 2, p. 640-653.

VAN HEEMST, H. D. J. 1986. Crop phenology and dry matter distribution. In: VAN KEULEN, H.; WOLF, J. (Ed.). Modelling of agricultural production: weather, soils and crops. Wageningen: PUDOC, p. 27-40.

VILLA NOVA, N. A.; BARBIERI, V. 1991. Viabilidade da cana-de-açúcar na região de Piracicaba, SP. In: CURSO DE AGRICULTURA IRRIGADA, 1991, Piracicaba. Piracicaba: ESALQ, p. 7682.

WALDRON， J. C.; GLASZIOU， K. T.; BULL, T.A. 1967. The physiology of sugarcane. IX Factors affecting photosynthesis and sugar storage. Australian Journal of Biological Sciences, Melbourne, v. 20, p. 10431052.

ZHOU, M.; SINGELS, A.; SAVAGE, M. J. 2003. Physiological parameters for modeling differences in canopy development between sugarcane cultivars. Proceedings of the South African Sugar Technologists Association, Durban, v. 7, p. 610-621. 